\title{
Le silence de l'orateur romain : signe à interpréter, défaut à combattre
}

\author{
Charles GUÉRIN \\ Université Paul Valéry - Montpellier III \\ Institut universitaire de France
}

\section{Introduction}

En décrivant les conditions idéologiques qui, d'après lui, ont stimulé le développement de l'art oratoire à Rome, Tacite, dans son Dialogue des orateurs, attribue deux causes bien distinctes à la compétition qui fait s'affronter les orateurs républicains : la gloire liée à la qualité d'homme éloquent d'une part (disertum haberi pulchrum et gloriosum), la honte de rester silencieux de l'autre (mutum et elinguem uideri deforme habebatur) ${ }^{1}$. Aux praemia que procure l'éloquence maîtrisée, s'ajoutent la necessitas et le rubor qui contraignent le citoyen un tant soit peu ambitieux à prendre la parole en public ${ }^{2}$. Nul ne pourra conquérir et conserver une place dans la cité autrement que par ses discours, et le silence, si l'on suit cette analyse, fait courir le risque de la relégation symbolique, mais aussi institutionnelle. Le patronus qui ne parviendrait pas à défendre ses clients manquerait à ses devoirs de fides et de diligentia ${ }^{3}$, perdrait toute existence véritable dans l'espace judiciaire ${ }^{4}$ et, du moins dans le monde de la République, ne pourrait plus jouer un rôle central dans la vie de la cité5.

Derrière cette description profondément politique du silence ${ }^{6}$, résonne également un lieu commun des traités et des manuels rhétoriques, selon lequel l'être humain, par opposition aux bêtes, est avant tout défini par sa capacité à parler? ${ }^{7}$ Politiquement et socialement déconsidéré, le silence dans l'espace public prend, à la lumière de ces textes, une coloration éthiquement

1. Tac., dial. 36, 8 .

2. Tac., dial. 36, 8-37, 1 : Ita ad summa eloquentiae praemia magna etiam necessitas accedebat [...]. Ergo non minus rubore quam praemiis stimulabantur [...].

3. Sur ces devoirs de l'orateur, cf. David 1992, p. 83-98.

4. Il existe également une forme d'assistance silencieuse, celle apportée par l'aduocatus qui soutient un citoyen par ses conseils et sa présence, et non par son discours. Il ne s'agit pas là d'un statut permanent, mais uniquement d'une situation temporaire : l'aduocatus dans une affaire peut être pleinement patronus dans une autre (cf. David 1992, p. 49-52). C'est le silence permanent qui, d'un point de vue symbolique, entraînerait de véritables difficultés.

5. Dans l'espace politique républicain, l'exercice des magistratures ne peut se concevoir indépendamment de l'exercice de la parole, et suppose en premier lieu la maîtrise de l'éloquence judiciaire. Sur ce phénomène, cf. David 1992, p. 591-641.

6. Le développement de dial. 36-37 est mené par le personnage de Secundus, pour qui la nature et la qualité de l'éloquence dépendent directement des conditions politiques dans lesquelles s'exerce cette dernière. Secundus s'intéresse donc à l'ancrage historique des pratiques oratoires, et décrit dans ce passage des réalités révolues. On constatera cependant que l'impératif éthique de la prise de parole - et la honte qui s'attache au silence - est toujours valide aux yeux de Quintilien dans le contexte du principat (cf. infra, p. 21). Sur la réflexion que Tacite consacre à l'évolution des pratiques oratoires, cf. en particulier Levene 2004, p. 157-200.

7. Cic., inv. I, 5. Voir également Cic., de orat. I, 32 ; Quint., inst. II, 16, 13-15 ; V, 11, 34 ; XII, 1, 2. Cette topique occupe une place essentielle dans les éloges de l'éloquence, et se rencontre déjà chez Isocrate, Nic. 5-6. 
négative : l'homme qui échoue dans le domaine qui définit son humanité est nécessairement un être dégradé. Salluste reprend à son compte cette topique en assimilant le silence de l'homme à celui de la bête brute menée par sa natura et dépourvue de raison ${ }^{8}$. L'absence de parole, caractéristique de l'irrationalité, fait alors soupçonner chez l'individu qui garde le silence moins une sorte de sagesse ${ }^{9}$ qu'un défaut naturel, une incapacité technique ou une forme de malhonnêteté.

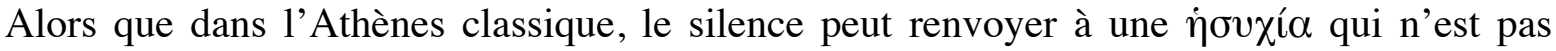
nécessairement négative et qui peut fournir un fondement efficace à la présentation de soi l'orateur se dépeignant comme un citoyen tranquille que les circonstances contraignent à sortir de sa réserve naturelle ${ }^{10}-$, Rome, en contexte oratoire et politique du moins, ignore ce type de valorisation. Dans la plupart des cas, l'orateur muet sera regardé comme un être incapable, stupide ou pervers. On touche ici du doigt la spécificité culturelle du silence ${ }^{11}$, dont le fonctionnement est tout aussi particulier et tout aussi propre à une civilisation donnée que, par exemple, celui du rire ${ }^{12}$, dans la mesure où son usage est régi par des codes implicites que la doctrine rhétorique ne parvient pas véritablement à énoncer ${ }^{13}$. Pour la théorie latine du discours, en effet, le silence est avant tout une marque d'échec, un risque que l'orateur doit éviter et un piège dans lequel il doit enfermer son adversaire. Réduire son contradicteur au silence représente en quelque sorte la victoire ultime dans l'affrontement oratoire ${ }^{14}$ et quand Cicéron s'enorgueillit de la qualité de son éloquence pathétique, c'est sa capacité à utiliser les passions pour contraindre ses adversaires à se taire qu'il met en avant :

\footnotetext{
Quo genere nos mediocres aut multo etiam minus, sed magno semper usi impetu saepe aduersarios de statu omni deiecimus. Nobis pro familiari reo summus orator non respondit Hortensius ; a nobis homo audacissimus Catilina in senatu accusatus ommutuit ; nobis priuata in causa magna et graui cum coepisset Curio pater respondere, subito assedit, cum sibi uenenis ereptam memoriam diceret ${ }^{15}$.

C'est grâce à ce genre d'éloquence ( $s c$. l'éloquence pathétique) que nous, tout médiocre que nous sommes, ou même moins encore, mais faisant toujours usage d'une grande vigueur, nous avons souvent fait complètement perdre pied à nos adversaires. Alors qu'il défendait un proche ( $s c$. Verrès), un grand orateur comme Hortensius n'a rien trouvé à nous répondre ; mis en cause par nous en plein Sénat, Catilina, le plus effronté des hommes, est resté muet ; alors qu'il avait commencé de nous répondre, dans une cause privée de grande importance, Curion le père s'assit brusquement en
}

8. Sall., Catil. 1, 1: Omnis homines qui sese student praestare ceteris animalibus, summa ope niti decet ne uitam silentio transeant ueluti pecora, quae natura prona atque uentri oboedientia finxit. Sur la lecture de silentio comme silence de l'individu et non comme silence à son sujet (sens actif et non passif), cf. Woodman 1973, p. 310 et Baker 1982, p. 801-802, qui discutent les parallèles possibles entre silentio, siletur (Catil. 2, 8), bene dicere $(2,1)$, celebrari $(8,3)$ et laudari $(8,5)$.

9. Cf. Valette-Cagnac 2004, p. 101.

10. Montiglio 2000, p. 131. Sur le topos du citoyen tranquille, cf. Carter 1986. Sur les tensions idéologiques opposant silence de la tranquillité et prise de parole active à Athènes cf. Guérin 2009, p. 40-61 et la bibliographie fournie.

11. Montiglio 2000, p. 3-6.

12. Le Goff 1989, p. 1

13. Sur les difficultés auxquelles se heurte la théorie rhétorique dans ses efforts pour formaliser les pratiques discursives et politiques, cf. Guérin 2011, p. 146-150.

14. Sur l'expression directe de la volonté de réduire l'adversaire au silence, cf. Cic., har. resp. 1-2 commenté par Cicéron en Brut. 316.

15. Cic., orat. 129 ; trad. A. Yon modifiée. Curio est également critiqué par Cicéron pour sa mauvaise mémoire : cf. infra, p. 14. 
disant qu'un philtre lui avait enlevé la mémoire.

À la fois objectif recherché par l'orateur et menace qui pèse sur lui, ce silence de l'échec ne doit pas être confondu avec les autres formes de silence qui peuvent apparaître dans le corpus rhétorique et oratoire. Proclamé ou mis en œuvre par une stylistique de l'allusion, du non-dit, de l'interruption et de l'aposiopèse, ce silence est essentiellement bavard et n'existe que parce qu'on le signale à l'auditoire. À l'évidence, la célèbre prétérition qu'emploie Cicéron dans la Première Catilinaire est moins silencieuse qu'elle ne le dit, puisqu'une telle figure insiste précisément sur ce qu'elle prétend taire ${ }^{16}$. Sénèque le Père fournit sur ce point une clé d'interprétation utile. D'après lui en effet, la déclamation peut faire usage de couleurs $\left(\right.$ colores $^{17}$ ) jouant sur des sous-entendus (controuersiae quae suspiciose dicendae erant ${ }^{18}$ ) et exploitant de ce fait le silence et les interprétations qu'il suscite. Le déclamateur Junius Othon était particulièrement habile à les manier :

Solebat hos colores, qui silentium et significationem desiderant, bene <dicere> ; itaque et hanc controuersiam hoc colore dixit, tamquam in emendationem abdicatorum et reconciliationis causa faceret $^{19}$.

Il excellait généralement dans les couleurs qui demandent silence et allusions. Par conséquent, il traita également cette controverse en usant de la couleur selon laquelle le but de l'homme ( $s c$. le fils du pauvre) avait été de corriger les fils reniés et d'oeuvrer à la réconciliation.

Le silence apparaît dans ce cas sous une forme technique, où il est mimé, signifié et souligné dans un but évidemment expressif ${ }^{20}$. Il peut alors être théorisé par la rhétorique ancienne, essentiellement comme un appareil stylistique visant à donner les moyens de manifester l'absence de la parole - par incapacité à s'exprimer ou par refus de le faire ${ }^{21}$. À l'inverse, le silence auquel nous nous intéresserons ici n'est ni proclamé, ni mis en scène, mais s'apparente davantage à un fait brut : il n'est pas choisi pour faire entendre à demi-mot ce qui doit l'être,

16. Quod ego praetermitto et facile patior sileri, ne in hac ciuitate tanti facinoris immanitas aut exstitisse aut non uindicata esse uideatur. Praetermitto ruinas fortunarum tuarum, quas omnis impendere tibi proxumis Idibus senties [...] (Cic., Catil. I, 14).

17. Chez Sénèque le père, le color désigne l'interprétation des faits qui orientera la narration et la démonstration dans une déclamation. Sur cette notion, cf. Fairweather 1981, p. 166-178 ; Montefusco-Calboli 2003, p. $115-117$; Lévy 2006, p. 185-199.

18. Contr. II, 1, 34 .

19. Sen. rhet., Contr. II, 1, 37 (en l'absence de mention contraire, les traductions sont les nôtres). La controverse porte sur un pauvre qu'un homme riche, qui vient de déshériter ses trois fils, souhaite adopter. Ce pauvre ayant refusé l'adoption, son propre père le déshérite.

20. E. Valette-Cagnac (2004, p. 103) remarque ainsi, à propos de la figure de reticentia théorisée par Cicéron, « le caractère énoncé, désigné, de ce silence » stylistique.

21. Sur cette stylistique du silence et ses effets, cf. Bardon 1943, p. 102-120 ; Dalimier 1989, p. 27-32 ; ValetteCagnac 2004. On constate un intérêt croissant pour les différentes formes de " rhétorique du silence », où le silence, sans être nécessairement signalé par un moyen stylistique, est étudié comme un moyen de communication à part entière. Sur cette analyse de la fonction expressive du silence - qui ne recoupe pas notre étude de sa valeur pratique, symbolique et technique dans le cadre de l'éloquence romaine -, on se reportera en particulier à Elissalde 1997 et Glenn 2004. La réflexion sur le silence peut également aboutir à une valorisation de celui-ci à partir d'une mise en question du rapport entre parole et vérité, comme c'est le cas chez Augustin (cf. Mazzeo 1962, p. 182-183 et 187-195). Cet axe d'analyse, qui ouvre de larges perspectives d'interprétation philosophique, sort néanmoins du cadre intellectuel de l'éloquence et de la rhétorique classiques à Rome et ne sera pas abordé dans cet article. 
mais imposé ou subi. Il ne s'agit plus alors du silence de l'intelligence, de l'allusion et de la stratégie, mais du silence de l'échec, du blocage et de la disqualification, un silence «véritable» par opposition à un silence « joué ».

Dans les témoignages et les réflexions qui nous sont parvenus sur ce sujet par nature fuyant, il faut donc distinguer le silence conçu comme moyen d'expression et le silence effectif, puisque l'un et l'autre renvoient à des phénomènes entièrement différents. Cette distinction est moins aisée qu'il n'y paraît au premier abord. Elle repose avant tout sur le rapport de l'orateur à son public : contrairement au silence construit par le biais de la stylistique, le silence réel n'offre pas de sous-texte et ne dit rien de plus que ce qu'il est lui-même, soit l'arrêt ou l'absence du discours. À l'inverse du silence construit qui repose sur la complicité entre orateur et auditeur, le silence véritable entraîne une rupture dans la relation établie entre orateur et auditoire : on peut donc considérer qu'il y a véritablement silence dès que ce qui était attendu par le public n'est pas exprimé par l'orateur, sans que cette absence suggère d'ellemême une interprétation qui vienne la compenser.

Dans l'échange oratoire, le véritable silence est avant tout un manque qui suscitera l'étonnement, l'embarras, le rire voire la fureur du public - soit, dans chaque cas, une réaction qui met fin aux conditions correctes et normales de l'énonciation. C'est ce silence brutal et destructeur que nous tenterons d'analyser ici, en nous appuyant sur un corpus rhétorique, oratoire et déclamatoire couvrant les Irs siècles avant et après J.-C. et englobant Cicéron, Sénèque le Père, Quintilien ainsi que le recueil d'exempla de Valère Maxime ${ }^{22}$. Nous poursuivrons deux objectifs principaux. Le premier consiste à montrer que ce silence « concret » est toujours interprété et que, à la différence du silence factice, cette interprétation ne peut être contrôlée par l'orateur : elle nécessairement périlleuse. Le silence, même s'il n'est rien d'autre que lui-même et qu'il est dépourvu d'intention signifiante, se lit et s'analyse dans le contexte de la performance oratoire : il devient alors un objet d'affrontement sur la scène du discours. En second lieu, nous entendons démontrer que, face à ce risque de l'interprétation, la rhétorique adopte un autre point de vue où le silence, loin de traduire un sens quelconque, est avant tout un échec, le plus lourd qui soit, qu'il faut absolument prévenir. On assiste alors à une différenciation très nette des analyses pratiques et théoriques du silence oratoire, différenciation qui aboutit, chez Quintilien, à la mise en place d'une technique, mais aussi d'une éthique, visant à combattre le silence.

\section{L'interprétation argumentative du silence}

Le silence de l'adversaire, constitue un événement que l'on interprète avec plus de facilité encore qu'une parole. Le silence s'offre à l'analyse, et peut bien sûr donner lieu à un affrontement, chacun tentant d'imposer au mutisme de l'orateur le sens qui lui est le plus favorable. Ainsi, dans le cadre d'un débat sénatorial où les discours appellent une réponse -

22. Les notions mises en jeu dans notre étude ne montrant aucune évolution chronologique notable, cet article adopte une approche synchronique et synthétique. Nous ferons par ailleurs ponctuellement appel à des textes plus éloignés du monde de l'éloquence. 
contrairement aux discours comiciaux où le silence du public est normal et attendu ${ }^{23}-$, le silence des orateurs qui composent l'assistance doit être pesé.

Dans une lettre à son frère Quintus, Cicéron fait le récit de l'altercation qui opposa Cn. Cornelius Lentulus Marcellinus à Publius Rutilius Lupus, le 10 décembre 57. Rutilius se réjouissait du silence complet dans lequel les sénateurs avaient accueilli le discours qu'il avait consacré à la question de l'ager Campanus, en l'absence de Pompée : à ses yeux, c'était là un signe d'approbation évident (ex praesenti silentio quid senatus sentiret se intellegere dixit), et il ne restait plus qu'à lever la séance. Marcellinus refusa qu'il en soit ainsi, et contesta l'interprétation de Rutilius. Ni approbation ni désapprobation du fond du propos, le mutisme du Sénat avait un sens bien particulier : il signalait d'après lui le refus d'engager un débat sur cette question avant le retour de Pompée ${ }^{24}$. Trois interprétations concurrentes étaient donc possibles, et c'est bien sûr ce caractère malléable et ouvert qui fait du silence une occasion de lutte entre orateurs.

Dans le cadre judiciaire, le silence est présenté en premier lieu comme une forme de triomphe de la vérité : celui qui n'a rien à répondre vient confirmer la validité des affirmations de son adversaire. Ce mutisme se rencontre chez tous les intervenants du procès et, bien que ceux-ci - accusés, témoins, patroni - s'inscrivent dans des situations d'énonciation différentes, l'interprétation de leurs silences sera sensiblement la même : accusés et témoins nous fournissent ainsi des modèles d'analyse applicables à l'orateur. Ainsi, le témoin qui reste silencieux durant son interrogatio trahit qu'il n'a rien à opposer aux questions qu'on lui pose ${ }^{25}$. Dans le cas de Roscius le comédien, les témoins qui restent sans voix confirment malgré eux qu'il n'existe aucune preuve matérielle incriminant le client de Cicéron :

Datam <pecuniam> non esse Fannius confitetur, expensam latam non esse codices Fanni
confirmant, stipulatam non esse taciturnitas testium concedit ${ }^{26}$.

Fannius avoue que la somme n'à pas été remise, les livres de Fannius prouvent qu'il n'en a pas été passé écriture, par leur silence, les témoins conviennent qu'elle n'a pas été stipulée.

23. Dans le cadre des discours au peuple, le silence du public constitue une marque essentielle d'approbation : le silence du peuple vaut jugement positif (sur la conception cicéronienne du jugement de la foule, cf. Schenkeveld 1988). Plus généralement, le peuple n'est pas supposé réagir au discours du magistrat durant les contiones : ses réponses, quand elles ont lieu, sont perçues comme une rupture de la situation normale d'énonciation et comme les prémisses potentielles d'une explosion de violence physique. Sur cette règle imposant le silence au public et les manipulations qu'elle pouvait faire naître, cf. Moreau 2003. De façon plus générale, sur les interactions orateur-public, cf. Aldrete 1999 ; Balbo 2007.

24. Cic., ad Q.fr. 2, 1, 1. Pour d'autres exemples de jeux d'interprétation de ce type, cf. Cic., Catil. I, 21 ; Caecin. 21 ; Sest. 40.

25 La parole du témoin se présente comme une réponse aux questions posées respectivement par l'accusateur et le défenseur dans la dernière phase du procès (sur la forme de cet échange, cf. Steck 2009, p. 80-83). Le refus de répondre valide la démonstration que mène l'orateur par son questionnement. Ce silence fournit alors un modèle d'interprétation pour celui de l'accusé - qui, lui, n'est pas tenu de répondre - et de l'orateur.

26. Cic., Q. Rosc. 14 ; trad. J. Humbert. 
La question laissée sans réponse fait éclater la vérité. Le processus est proche de celui consistant à tourner un adversaire en ridicule : là encore, le but ultime de la manœuvre consiste à faire taire l'adversaire en révélant au public une vérité qui lui avait échappé, mais qui, une fois soulignée, déclenche le rire. Le De oratore rappelle ainsi comment César Strabon avait pu affaiblir son adversaire Helvius Mancia en pointant sa ressemblance avec un portrait caricatural de Gaulois exposé près du forum ${ }^{27}$. Victime du rire déclenché par César, Mancia ne sut pas répliquer ${ }^{28}$. Le processus est semblable à celui décrit dans le Pro Roscio Comoedo : c'est le dévoilement d'une forme de vérité - ici, celle de la ressemblance à un type physique non romain ${ }^{29}$ - qui prive l'individu de toute possibilité de répartie. La parole ne peut rien contre l'apparence rendue soudain visible et lisible aux yeux de tous ${ }^{30}$. La forme de domination que traduisent les phénomènes de ce genre se retrouve de façon plus claire encore dans les situations où un orateur fait usage de son autorité pour parasiter la situation d'énonciation. Lorsque Scipion, accusé par le tribun Naevius, fit cesser le procès en emmenant le public à sa suite rendre grâce aux dieux de sa victoire sur Hannibal, c'est la vérité de la situation - en l'occurrence l'ineptie et le scandale de l'accusation - qui éclata aux yeux de tous, rendit la parole inutile et réduisit l'accusateur au silence ${ }^{31}$.

Le silence de l'accusé et de son patronus peuvent également apporter une preuve supplémentaire de la véracité des accusations : le mutisme est alors présenté comme une forme d'acquiescement ${ }^{32}$. Dans le cadre fictionnel de la Seconde action contre Verrès, le silence du préteur vient confirmer le récit des témoins et les affirmations de l'accusateur, comme dans l'épisode célèbre de l'incendie de Lampsaque :

Cum igitur quae causa illius tumultus fuerit testes a nobis producti dixerint, ipse celarit, nonne causam hanc quam nos proposuimus cum illorum testimonia tum istius taciturnitas perpetua

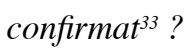

Puisque les témoins que nous avons cités ont fait connaître la cause de cette émeute, et que lui refuse de la révéler, ne faut-il pas considérer que leurs dépositions et le silence obstiné de l'accusé corroborent également l'affaire que nous avons exposée?

De confirmation, le silence en vient logiquement à être présenté comme un aveu ${ }^{34}$. Dans ce cas, l'argumentaire ne fait que reprendre des prescriptions traditionnelles que l'on retrouve dès les analyses du De inuentione, où Cicéron fait du silence de l'adversaire un constituant essentiel $\mathrm{du}$ raisonnement par inférence. Que l'adversaire reconnaisse explicitement la justesse de

\section{Cic., de orat. II, 266.}

28. Le De oratore (de orat. II, 262) évoque également un phénomène plus brutal encore où la réponse à une première attaque ayant déclenché les rires entraîne une répartie cinglante et un redoublement du chahut : l'orateur (L. Aelius Lamia) est alors réduit au silence d'autant plus violemment qu'il a prétendu répondre.

29. L'anecdote revêt également un sens politique et social qui n'entre pas dans notre propos ici : cf. sur ce point Guérin 2009a, p. 231-233.

30. L'analyse du rire comme dévoilement, déjà présente chez Aristote (Cf. Guérin 2009, p. 203), est centrale dans le De oratore : cf. sur ce point Guérin 2011, p. 179-180.

31. Gell. IV, 18, 3-5.

32. Sur ce mécanisme, cf. Perelman et Olbrechts-Tyteca 1958, p. 145-146.

33. Cic., Verr. II, 1, 80.

34. Cf. Cic., Sest. 40. De façon générale, J.-M. David souligne l'impossibilité pratique de l'aveu à Rome à la fin de la République et explique par ce biais le lien établi entre silence et reconnaissance du crime (David 1986, p. 73-75; David 1992, p. 96-97). 
l'argument qu'on lui oppose ou qu'il se taise, la démonstration peut être conclue de la même manière ${ }^{35}$ :

Si concedetur, concludenda est argumentatio. Si tacebitur, elicienda responsio est aut, quoniam taciturnitas imitatur confessionem, pro eo, ac si concessum sit, concludere oportebit argumentationem $^{36}$.

S'il la concède, il faudra tirer la conclusion de l'argumentation. S'il refuse de parler, il faudra lui arracher la réponse ou bien, puisque le silence est une forme d'aveu, conclure l'argumentation, exactement comme si elle se trouvait admise.

Faisant écho aux silences de Verrès, Hortensius lui-même n'aurait pas su répondre au discours de Cicéron et aux témoignages qu'il avait présentés ${ }^{37}$.

Le silence, dans ce premier cas de figure, marque en quelque sorte l'irruption du réel - ou de ce que l'orateur veut présenter comme tel - dans l'espace de l'argumentation : le discours s'interrompt ou ne débute même pas car il n'a aucun poids face à la réalité qu'il cherche à nier. Le Pro Flacco illustre parfaitement le phénomène. Au cours de son plaidoyer, Cicéron raconte comment un accusé volubile à l'excès - Héraclide de Temnos, un homo ineptus et loquax (Flacc. 42) - s'arrêta net et sombra dans le silence lorsqu'on lui opposa un témoignage et des pièces irréfutables :

Sed cum se homo uolubilis quadam praecipiti celeritate dicendi in illa oratione iactaret, repente testimoniis Fufiorum nominibusque recitatis homo audacissimus pertimuit, loquacissimus obmutuit $^{38}$.

Mais tandis qu'avec la facilité qui est la sienne, il se faisait valoir dans son discours et se laissait porter par le torrent de ses paroles, soudain, entendant lire la déposition des Fufius et leurs titres de créance, notre effronté fut frappé de terreur, cet extraordinaire bavard resta muet.

La preuve extrinsèque réduit au silence le discours creux, dans une forme de victoire du fait sur le verbe mensonger ${ }^{39}$. Les mises en scène artificielles qui échouent peuvent également aboutir au silence : ainsi, un orateur habituellement éloquent (facundus), qui avait prévu d'embarrasser l'adversaire en faisant circuler jusqu'à lui, de bras en bras, un nourrisson qui était prétendument sa sœur, fut contraint d'aller rechercher l'enfant en silence et de cesser abruptement son développement pathétique lorsqu'il s'avéra que l'adversaire, conscient de la manoeuvre, s'était opportunément éclipsé ${ }^{40}$. Là encore, c'est bien le réel et ses contraintes qui viennent invalider le discours et en souligner le caractère inutile.

À ce premier type de silence - le silence de celui qui n'a rien à répondre, ou dont le développement est paralysé par les circonstances - s'en ajoute un deuxième, dont l'interprétation est plus subtile. Dans ce cas, le silence ne doit pas être interprété comme une

35. On retrouve dans les Controuersiae de Sénèque le Père le même usage topique du silence comme aveu, cf. Contr. X, 2, 6 .

36. Inv. I, 54 ; trad. G. Achard.

37. C'est ce qu'affirme Cicéron en orat. 129 (cité supra, p. 2) et ce qu'il laisse entendre en Verr. II, 1, 3, 20 et 31 ; 2, 2, 192. Il reste cependant peu probable qu'Hortensius soit véritablement resté silencieux : cf. inst. X, 1, 22-23, qui mentionne son Pro Verre, et la démonstration d'Alexander 1976.

38. Cic., Flacc. 48 ; trad. A. Boulanger modifiée.

39. Cf. également l'anecdote rapportée par Val. Max. V, 3, ext. 3.

40. Inst. VI, 1,39. 
étape dans l'interaction qui oppose les adversaires, mais comme un signe de l'état intérieur de l'individu qui reste muet. L'incapacité à s'exprimer constitue alors une manifestation physique de la culpabilité ou, plus exactement, de la crainte et du remords qu'elle crée ${ }^{41}$. Cette incapacité présente différents degrés, qui vont du bredouillement au silence complet ${ }^{42}$.

Un premier exemple judiciaire du phénomène apparaît dans les Verrines, où le publicain Carpinatius, complice du préteur, reste mutus et exanimatus metu conscientiaque peccati ${ }^{43}$ alors que Cicéron saisit les archives de la société qu'il dirige et l'interroge sur les détournements commis. Une réaction de ce type peut également se rencontrer en situation oratoire, et l'exemple le plus significatif reste celui de Catilina. Malgré son immoralité, la conscience de ses crimes le réduisit au silence ${ }^{44}$, alors même qu'il aurait pu prendre la parole et se défendre :

\begin{abstract}
Cum ille homo audacissimus conscientia conuictus primo reticuisset, patefeci cetera ; quid ea nocte egisset, quid in proximam constituisset, quem ad modum esset ei ratio totius belli descripta edocui. Cum haesitaret, cum teneretur, quaesiui quid dubitaret proficisci eo quo iam pridem pararet $[. . .]^{45}$.

Comme il gardait d'abord le silence - il ne recule devant absolument rien, mais sa conscience le condamnait -, j'ai exposé tout le reste au grand jour et j'ai raconté ce qu'il avait fait cette nuit-là, ce qu'il avait prévu pour la suivante, comment il avait tracé le plan de la guerre dans le détail. Il bafouillait, je l'avais acculé : alors je lui demandai pourquoi il hésitait à se rendre là où il avait prévu de partir depuis longtemps $[\ldots]$.
\end{abstract}

Le passage montre par ailleurs que ces deux premiers types de silence sont complémentaires : Catilina, en effet, n'est réduit au silence par sa conscience que pour l'être à nouveau par le discours de Cicéron qui le harcèle de ses questions. Le signe éthique du silence est ainsi renforcé et redoublé par le silence dans l'interaction ${ }^{46}$.

Face à ces individus contraints au silence par leurs adversaires ou par leur propre culpabilité, on rencontre quelques exemples d'orateurs ayant délibérément choisi de se taire. Dans les rares cas où elles sont présentées de façon positive, les raisons de ce silence sont identiques à celles invoquées par les orateurs mettant en scène leur propre refus de s'exprimer ${ }^{47}$. Néanmoins, c'est le plus souvent comme une marque de malhonnêteté qu'est interprété le mutisme délibérément

41. Cf. Catil. III, 18 qui place le silence des conjurés sur le même plan que leur teint, leurs yeux, leur regard et leur physionomie.

42. Le De domo (dom. 139-140 ; voir également le récit fictionnel en dom. 134-135) fait un large usage de cette thématique dans son récit de la dedicatio menée par un Clodius pleinement conscient de l'illégalité qu'il est en train de commettre et qui, par conséquent, s'embrouille et bégaie (mutus). Dans le cadre du De domo, cette caractérisation des adversaires ne sert pas qu'un but d'invective et se situe en réalité au cœur de l'argumentaire : la consécration ayant été mal prononcée, celle-ci est bien évidemment invalide. Pour éclairant qu'il soit, l'exemple se situe néanmoins en dehors du domaine oratoire.

43. Verr. II, 2, 189.

44. Les Verrines font déjà un usage antiphrastique de ce lieu, en soulignant que le silence et l'absence de Verrès à son procès auraient pu marquer une sorte de conscience et de pudeur chez l'accusé : cf. Verr. II, 1, 2.

45. Catil. II, 13.

46. La même coïncidence d'une paralysie issue de la situation et d'une aphasie due au poids de la conscience se rencontre, en dehors du contexte oratoire, chez Cethegus, durant la même conjuration : cf. Cat. III, 10.

47. Cf. Bardon 1943. Il s'agit en particulier du respect des convenances (Catil. I, 14) et du refus de l'obscénité (inst. VIII, 3, 39). La crainte n'est pas mentionnée dans le corpus comme motif d'un véritable silence chez un orateur, mais c'est elle qui empêche de nombreuses cités siciliennes de témoigner de ce qu'elles ont subi (Verr. II, 5, 57). Val. Max. IV, 7, 1 offre un contre-exemple éclairant à propos de C. Blossius que la crainte aurait normalement dû réduire au silence, mais qui persiste à répondre aux questions de C. Laelius devant qui il tentait de justifier son engagement auprès des Gracques. 
choisi : il est alors présenté soit comme une manipulation délibérée, soit comme le résultat d'une corruption.

Ainsi, en gardant le silence sur un point essentiel du procès, les accusateurs de Flaccus dissimulent ce que le jury serait en droit d'entendre et masquent la véritée ${ }^{48}$. Le silence de l'accusateur est alors synonyme de mensonge et doit pour cela être traqué avec soin. Mais il peut également signaler la collusion. S'il était choisi comme accusateur de Verrès, nous dit Cicéron, Caecilius s'efforcerait de taire (nulla mentio fiet; silebitur) les crimes du préteur qu'il cherche à protéger ${ }^{49}$. De la même manière, Cicéron dénonce la collusion manifeste entre l'accusateur Lentulus et l'accusé Gabinius, face à qui Lentulus a soudain perdu toute son éloquence (nihil accusatore Lentulo subscriptoribusque eius infantius ${ }^{50}$ ) : l'accusatorum incredibilis infantia a d'ailleurs été comprise par tous comme le résultat d'une prévarication ${ }^{51}$.

Le silence d'un orateur peut donc être interprété de trois manières différentes au cours de l'échange oratoire : soit la parole se trouve paralysée par la vérité qu'on lui oppose ; soit elle est éteinte par la conscience du crime ; soit elle est volontairement étouffée par la malhonnêteté ou la prévarication. Face à ces interprétations, aucune analyse positive du silence réel n'est jamais formulée dans notre corpus - à l'exception d'un exemplum de Valère Maxime portant sur un témoin gardant le silence dans une affaire de mœurs ${ }^{52}$. Dans tous les cas, ce silence est circonstanciel : le mutisme qui survient dans un échange n'informe en rien sur les compétences d'un orateur, mais uniquement sur l'affaire et le rapport que cet orateur entretient avec elle. C'est une posture exactement inverse qu'adoptera la doctrine rhétorique en substituant à l'observation du sens du silence une enquête portant sur ses cause naturelles et techniques. Le but, pour la théorie du discours, n'est en effet plus d'enregistrer ce silence pour l'interpréter, mais bien de fournir les moyens de l'éviter ou, s'il survient, de le surmonter. L'analyse du mutisme comme signe laisse alors la place à une analyse du silence comme simple défaut.

\section{L'interprétation technique du silence}

On doit remarquer en premier lieu que cette approche étiologique et technique du silence n'est pas réservée à la théorie du discours. Elle apparaît également au sein du corpus oratoire,

48. Flacc. 6 : muta est huius temporis accusatio. Les protestations d'exhaustivité qui se rencontrent dans le corpus judiciaire cicéronien (cf. par exemple Cluent. 1) doivent alors s'interpréter comme des manifestations d'intégrité. L'orateur, en affirmant qu'il dira tout, souligne surtout qu'il ne dissimulera rien : cf. Valette-Cagnac 2004, p. 108.

49. Cic., diu. in Caec. 32 ; trad. H. de la Ville de Mirmont modifiée.

50. ad Q.fr. 3, 4, 1 .

51. Att. 3, 18, 1 ; ad Q.fr. 3, 4, 1 .

52. Val. Max. VI, I, 7. Il s'agit du fils de M. Claudius Marcellus, qui contribua à la condamnation du tribun Scantinius qui lui avait fait des avances sexuelles. Cité comme témoin contre lui, il garda le silence (Constat iuuenem [...] defixo in terram uultu perseueranter tacuisse uerecundoque silentio plurimum in ultionem suam ualuisse) sous l'effet d'une uerecundia qui lui interdisait de rendre compte des faits, alors même que le cadre de l'interrogatio lui imposait de répondre (cf. supra p. 5, n. 25). Sur cette uerecundia qui empêche le sujet d'agir et de parler - comme le fait l'infans pudor horatien en sat. I, 6, 57 -, cf. Thomas 2007, p. 416-423 et, sur le pudor paralysant, ibid., p. 331-336. Le complet mutisme du jeune Marcellus est donc à la fois réel (le jeune homme est véritablement empêché de parler) et signifiant, puisqu'il est interprété par les juges et perçu comme un signe de la culpabilité de Scantinius (voir également Plut., Marcel. II, 5-8 où les larmes et la rougeur du jeune homme, plus que son silence, emportent la décision des juges). Bien qu'il soit valorisé, on doit remarquer que ce silence reste un marqueur de ueritas au même titre que le silence condamnable des témoins confondus : dans les deux cas - et quel que soit le mécanisme qui conduise au mutisme -, c'est la réalité du fait qui impose le silence. 
dans des passages qui servent à décrire les tares de l'adversaire et dont la portée n'est plus démonstrative mais éthique. Le silence de Pison, dont personne, d'après l'orateur, n'avait entendu la voix avant son consulat et qui avait su dissimuler le stupor et la debilitas linguae dont il souffrait, est exploité par Cicéron dès le début de la réponse qu'il adresse à son adversaire, en plein Sénat, en septembre 55 :

Pauci ista tua lutulenta uitia noramus, pauci tarditatem ingenii, stuporem debilitatemque linguae. Numquam erat audita uox in foro $[\ldots]^{53}$.

Nous n'étions pas nombreux à connaître tes vices répugnants, ta lenteur intellectuelle, ta manière de parler incompréhensible et embarrassée. Jamais ta voix ne s'était fait entendre sur le forum [...].

Cette description, qui relève pour l'essentiel de l'invective ${ }^{54}$, fait apparaître un silence oratoire qui ne serait plus conjoncturel et temporaire, lié à la faute commise ou au débat lui-même, mais qui représenterait au contraire une caractéristique durable de l'individu incapable de parler. Dans ce cas, le silence n'est pas le résultat des circonstances mais un élément définitoire de l'identité d'un orateur.

Quintilien rappelle que Cicéron était prompt à critiquer l'infantia de ceux qui s'opposaient à lui, et que ce reproche suscitait efficacement la haine du public ${ }^{55}$. L'infantia désigne tout d'abord l'absence de parole chez l'enfant ${ }^{56}$ et, plus fréquemment, la première période de l'enfance précédant l'acquisition du langage ${ }^{57}$. Appliqué à l'adulte, il peut tout aussi bien traduire une aphasie d'origine physique qu'une incapacité technique. L'homme infans peut-être silencieux au sens plein ${ }^{58}$ ou - et c'est le cas le plus général - peu habile à s'exprimer : l'infantia désigne alors l'incompétence oratoire ${ }^{59}$. L'adjectif elinguis présente le même type d'ambiguïté puisqu'il possède également un sens fort (qui garde le silence ${ }^{60}$ ) et un sens faible (inhabile $\left.{ }^{61}\right)$. Le problème du silence surgit véritablement quand un individu physiquement apte à parler se révèle tellement malhabile qu'il ne parvient même plus à bredouiller, mais finit par se taire. L'infantia du malhabile devient alors la marque d'une incapacité totale, celle que dénonce Quintilien lorsqu'il critique les orateurs qui mettent sous les yeux du public un tableau représentant le fait incriminé parce qu'ils ne parviennent pas à le décrire par la parole ${ }^{62}$. Le reproche peut également porter sur des propos si incompréhensibles qu'ils équivalent au silence d'un non-discours, comme le sont les harangues de Rullus ${ }^{63}$ dont personne, nous dit Cicéron, ne saisit un traître mot. Mieux vaut se taire, comme Cicéron le suggère à Antoine dans

\section{Cic., Pis. 1.}

54. Sur les invectives contre Pison, cf. DeLacy 1941. Sur l'usage cicéronien de l'invective, cf. Corbeill 2002.

55. Quint., inst. V, 13, 38, qui mentionne Rullus, Pison et Antoine.

56. ThLL vol. 7, 1, 2, col. 1350, A, 2 .

57. ThLL vol. 7, 1, 2, col. 1347, I, A, 2 ; col. 1347-1348, II, A-B ; col. 1350, B, 1.

58. ThLL vol. 7, 1, 2, col. 1346, I, A, 1, a.

59. ThLL vol. 7, 1, 2, col. 1347, I, A, 1, b ; col. 1349-1350, A, 1. Cf. Rhet. Her. II, 16 ; inu. I, 4 ; de orat. III, 142 (qui définit la notion), 198 ; orat. 55, 76,236; fin. I, 52 ; Cluent. 51.

60. ThLL vol. 5, 2, col. 390, 2, a.

61. ThLL vol. 5, 2, col. 390, 2, b. Il faut ajouter qu'elinguis sert également à désigner un orateur réduit au silence (cf. Flacc. 22) et non pas seulement gardant le silence, ce qui n'est jamais le cas de l'adjectif infans. Dans ce cas, il reprend en quelque sorte un sens physique : «privé de langue » (ThLL vol. 5, 2, col. 390, 1).

62. Inst. VI, 1, 32.

63. Cic., leg. agr. II, 13. 
la $3^{e}$ Philippique, que de tenir des propos auxquels personne ne comprend rien ${ }^{64}$ : le caractère inintelligible d'un discours apparaît par conséquent comme un préliminaire au silence total.

Logiquement, le fait d'être non infans ${ }^{65}$ ou non elinguis ${ }^{66}$ constitue dans le Brutus de Cicéron la première étape dans la gradation des qualités menant à l'éloquence : Metellus Celer, tout en étant non infans, n'est pas encore un orateur accompli ${ }^{67}$. L'infans est donc l'exact contraire du disertus $^{68}$, selon une opposition que Quintilien reprendra à son compte en reliant par la même occasion l'infans et l'imperitus, l'orateur sans expérience ${ }^{69}:$ le rhéteur suggère ainsi que ces différents cas - celui du semi-silence du bredouillement, du silence du discours incompréhensible ou du mutisme complet - ont des causes qui sont tout autant techniques que naturelles.

La dimension naturelle de l'infantia n'occupe pas une place centrale dans le corpus rhétorique latin. La doctrine se contente de constater l'inaptitude physiologique ou intellectuelle à la parole, et de souligner que la technique ne peut en aucun cas l'améliorer. Pour Cicéron, les préceptes et l'entraînement ne parviendront généralement pas à réduire le défaut dont souffrent les haesitantes lingua : à quelques exceptions près - dont fait partie Démosthène - jamais on ne pourra les compter au nombre des orateurs ${ }^{70}$. Moins analysé que constaté et dénoncé, le silence qui découle de l'incapacité naturelle est évoqué pour l'essentiel dans notre corpus dans des injures et des invectives. Ainsi Pison, dont personne ne comprend les élucubrations, est silencieux à la manière d'une souche ou d'un animal. L'ennemi de Cicéron est elinguis et, à ce titre, il n'est plus véritablement homme ${ }^{71}$ :

Cum hoc homine an cum stipite in foro constitisses, nihil crederes interesse : sine sensu, sine sapore, elinguem, tardum, inhumanum negotium, Cappadocem modo abreptum de grege uenalium diceres $^{72}$.

Qu'on se soit arrêté sur le forum auprès de cet homme ou auprès d'une souche, on n'y aurait pas vu la moindre différence : stupide, insipide, muet, borné, une chose sans éducation qu'on aurait prise pour un Cappadocien à peine arraché à son troupeau d'esclaves.

À travers cette invective, on aperçoit les vastes possibilités d'agression et de stigmatisation de l'adversaire qu'offre le lien traditionnel entre parole, rationalité et humanité ${ }^{73}$. L'haesitantia

64. Cic., Phil. III, 22. Des remarques du même type se retrouvent en dehors du contexte oratoire, par exemple chez Gell., I, 10, 2.

65. Brut. 77, 90, 108, 305.

66. Brut. 100. Le qualificatif est appliqué à Fannius, soupçonné d'avoir fait écrire ses discours par autrui.

67. Brut. 305.

68. Brut. 101. Le passage porte sur la facultas in dicendo de Fannius, que l'on peut juger par l'historia qu'il a écrite : Cicéron oppose alors comme deux extrêmes le style infans et le style disertus. Quoiqu'appliquée à l'écriture de l'histoire, la remarque peut être étendue au style oratoire dans la mesure où l'historia écrite vaut ici comme témoignage de l'éloquence de son auteur.

69. Inst. XI, 1, 21.

70. De orat. I, 115 : in oratorum numerum uenire non possunt. Dans le corpus cicéronien, la figure de Démosthène représente la victoire de l'effort et de la maîtrise technique sur la nature. Cf. sur ce point Guérin 2011, p. 120-122.

71. Cf. également Pis. 73-74 où Cicéron souligne que Pison est un asinus qu'on doit éduquer à coups de bâton plus qu'avec des paroles.

72. Cic., p. red. in Sen. 14 ; trad. P. Wuilleumier modifiée.

73. Le bégaiement et le mutisme «naturels » peuvent constituer, en dehors même du contexte oratoire, des causes 
linguae est naturellement liée au stupor de l'intellect ${ }^{74}$, la difficulté à penser - et en particulier à trouver des arguments ${ }^{75}$ - faisant écho à la difficulté à s'exprimer ${ }^{76}$. Le silence total, quant à lui, ramène à l'absence de rationalité caractéristique de l'animal ${ }^{77}$.

Pour devenir un véritable objet théorique, il faut que le silence ne soit plus abordé comme une réalité naturelle, mais qu'il soit relié à un défaut technique. Notre corpus offre plusieurs descriptions de ces défauts susceptibles de réduire l'orateur au mutisme. Ceux-ci n'ont bien sûr rien à voir avec la stupeur que causerait la vérité éclatante ou le remords, et beaucoup plus avec la paresse ou l'incompétence. Pour la doctrine en effet, la première cause technique du silence est l'impréparation. Trop d'orateurs, nous dit Cicéron dans le De oratore $^{78}$, se présentent au procès sans avoir étudié leur affaire, en particulier sans avoir interrogé leur client. Ils s'exposent de ce fait à un double péril, celui de la lenteur et celui de la sécheresse. L'orateur qui n'a pas préparé ses arguments se trouve contraint de réfléchir et de parler en même temps : son débit s'en trouvera nécessairement ralenti. Il s'attirera une réputation de lenteur intellectuelle (tarditas) et sera renvoyé dans la sphère du défaut naturel ${ }^{79}$ : la parole ralentie, qui ruine la concentration du public ${ }^{80}$, n'est pas éloignée du silence total et se rapproche du bégaiement ou de la confusion. Cette lenteur peut également affecter un orateur mal entraîné ou qui ferait un mauvais usage des techniques d'invention. Essayer un à un tous les lieux que

de raillerie et d'exclusion. Ainsi, la linguae titubantia de Claude (Suet., Claud. 30) - cause de honte pour son entourage (ibid. 4 ; Baroin 2010a, p. 54) - est l'un des objets centraux de l'Apocolocyntose (cf. Osgood 2007, p. 329-353). Sur le handicap comme facteur d'exclusion à Rome, cf. Garland 1995, en particulier p. 11-27 et Gardner 2002, p. 155-178. Le mutisme, s'il interdit au citoyen de tester et d'occuper les fonctions de juge (Gardner 2002, p. 159-167 ; Ducos 2010, p. 98-99) ne fait pas naître de débat dans nos sources lorsqu'elles évoquent les handicaps interdisant l'exercice des magistratures (Mommsen 1892, p. 143 ; Baroin 2010a, p. 49-68).

74. Cf. le personnage de Bambalio, Phil. III, 16. Contrairement au défaut naturel qui frappe l'élocution, la tarditas ingenii peut être combattue à force de diligentia : de orat. II, 147. Il faut noter que, dans le corpus auquel nous nous sommes intéressé, le défaut de stupor (rendu également par l'adjectif stupidus) désigne systématiquement l'incapacité à élaborer un discours correct et non l'incapacité à l'énoncer : cf. Contr. VII, 2, 14 ; 3, 8 ; IX, praef. 2 ; Suas. II, 21. Dans ce champ lexical, seul le verbe stupeo peut désigner l'aphasie (Contr. VII, praef. 4 ; inst. XII, 6, 5). De façon générale, le stupor désigne l'incapacité intellectuelle (Cic., har. resp. 5 ; Phil. II, 30, 65, 80 ; III, 22 ; VIII, 24), le manque de sensibilité sensorielle (Phil. II, 115 ; Cic., Tusc. III, 6), le trouble ou la passivité (Cic., parad. V, 37 ; Contr. II, 7, 1 ; VII, 1, 1 ; inst. XI, 3, 159) ou une immobilité excessive paralysant l'actio (inst. IX, 3, 101). L'adjectif hebes sert aussi à désigner la faiblesse intellectuelle (inu. I, 24 ; de orat. I, 248 ; II, 72 , 133 ; Brut. 246 ; orat. 216 ; Phil. X, 17 ; inst. I, 1, 2 ; IV, 2, 66 ; VII, 1, 48) ou sensorielle (Cic., Planc. 66 ; de orat. II, 356 à propos de la mémoire), sans implication quant à la capacité d'expression.

75. Tarditas: leg. I, 51 ; inst. II, 2, 23 ; tarditas ingenii : orat. 229 ; inst. I, 1, 1 ; tarditas excogitandi : Brut. 235.

76. Cf. Brut. 235 : la tarditas loquendi de Publius Lentulus répond naturellement à sa tarditas excogitandi. On peut également citer la cas traditionnel de Manlius Torquatus, relégué à la campagne par son père à cause de ses difficultés d'élocution (lingua impromptus ; cf. Liv. VII, 4, 4-6) : il est frappant de voir que Valère Maxime exploite cet exemplum en présentant Torquatus comme un homme stupide (hebetis atque obtunsi cordis ; Val. Max. VI, 9, 1) et non pas comme un bègue. Sur les implications idéologiques de cette description de Torquatus, cf. l'analyse de J.-M. David, op. cit., p. 322-324.

77. Cf. les oppositions dressées par Quintilien en inst. V, 11, 34 ; XII, 1, 2.

78. De orat. II, 101. Cf. également Contr. X praef. 2 ; inst. X, 7, 20-22 ; XII, 8, 4-6.

79. L'accusation de tarditas est présentée par le De oratore comme un stigmate permanent : de orat. I, 124-125. Cf. également inst. XI, 3, 52 (difficultas inueniendi).

80. Inst. XI, 3, 52 . 
l'on a pu envisager paralyse le développement ${ }^{81}$ : c'est une fois encore l'expérience et la préparation qui permettront à l'orateur d'exploiter ses arguments de façon naturelle, sans que son débit ait à en souffrir ${ }^{82}$.

Le second risque de l'impréparation ou de la préparation inadéquate est celui de la sécheresse ${ }^{83}$. Manquant de matière, l'orateur mal préparé n'aura pas de quoi fournir un discours complet ; manquant de ressources, il ne pourra mener ses développements jusqu'à leur terme. C'est avant tout dans sa dimension lexicale que cette difficulté est envigagée. L'inopia, qui désigne la pauvreté du vocabulaire dans l'ensemble de notre corpus, interdit à l'orateur de s'exprimer comme il le devrait ${ }^{84}$ : elle est conçue comme l'équivalent de la tarditas intellectuelle dans le domaine de l'elocutio ${ }^{85}$. Le rhéteur Cestius représente un cas extrême d'inopia qui, même s'il concerne un déclamateur et non un orateur, offre des clés d'interprétation à valeur générale. La pauvreté de son lexique ne lui permet pas d'exprimer correctement ses idées et le contraint parfois à arrêter net son développement :

\begin{abstract}
Soleo dicere uobis Cestium Latinorum uerborum inopia <ut> hominem Graecum laborasse, sensibus abundasse ; itaque, quotiens latius aliquid describere ausus est, totiens substitit, utique cum se ad imitationem magni alicuius ingeni derexerat ${ }^{86}$.
\end{abstract}

J'ai coutume de vous dire que Cestius, parce qu'il était grec, souffrait d'un manque de mots latins alors qu'il avait des idées en abondance. Aussi, toutes les fois qu'il osa se risquer dans une description un peu longue, il resta court, surtout lorsqu'il se proposait d'imiter quelqu'un qui avait du talent.

Le cas de Cestius est particulier tant par sa conséquence - l'arrêt brutal du propos - que par ses causes. Sénèque explique le silence auquel il est réduit d'une triple manière. En premier lieu, Cestius est grec : le latin n'étant pas sa langue maternelle, il souffre avant tout d'un manque d'usage. Par ailleurs, son défaut se manifeste tout particulièrement quand il tente d'imiter les sententiae d'un autre : l'inopia se traduit par une incapacité totale ou, à tout le moins, par une gaucherie dans la reformulation. Enfin, ces échecs découlent d'une mauvaise évaluation de ses propres capacités, erreur qui pousse Cestius à se lancer dans des développements dont il n'est pas capable. Pour aiguë qu'elle soit, l'inopia du déclamateur n'est pourtant pas unique, comme le montre la précision qu'apporte Sénèque à propos du déclamateur-philosophe Papirius Fabianus dont il vante les descriptions en soulignant que jamais il ne les interrompait faute de ressources nécessaires :

[...] locorum habitus fluminumque decursus et urbium situs moresque populorum nemo descripsit abundantius. Numquam inopia uerbi substitit, sed uelocissimo ac facillimo cursu omnes res beata circumfluebat oratio ${ }^{87}$.

[...] l'aspect des lieux, le cours des fleuves, la situation des villes et les mours des peuples n'ont

81. Inst. $\mathrm{V}, 10,123$.

82. Inst. $\mathrm{V}, 10,125$.

83. Faute d'avoir préparé à l'avance des arguments efficaces et originaux, l'orateur, avant même d'être sec, court bien sûr le risque de la platitude et de l'inefficacité, mais ces défauts n'entrent pas dans le cadre de notre étude.

84. Fam. 4, 4, 1 ; Phil. X, 5 ; Brut. 202, 238, 221, 285 ; inst. II, 4, 4.

85. Cf. le parallèle dressé par Brut. 246 entre tardus sententiis et inops uerbis.

86. Contr. VII, 1, 27; trad. H. Bornecque modifiée.

87. Contr. II praef. 3 ; trad. H. Bornecque. 
jamais été décrits avec plus d'abondance. Jamais il ne resta court, cherchant un mot, mais tout était comme emporté dans le courant fécond de son style si rapide et si facile.

Si cette absence d'inopia uerbi est mentionnée comme une qualité, c'est bien que la sécheresse devait être fréquente et que Cestius constitue, de par ses origines et ses goûts, l'incarnation hyperbolique d'un problème général. Car indépendamment de la question ethnique ou du choix des modèles, c'est avant tout le travail à fournir par le praticien qui conditionnera sa capacité lexicale. De fait, dans son portrait de Fabianus, Sénèque rappelle l'entraînement permanent auquel celui-ci s'astreignait ${ }^{88}$.

La doctrine insiste à de nombreuses reprises sur cet aspect. C'est l'entraînement qui fournira la copia nécessaire à l'éloquence. En premier lieu vient l'écriture ${ }^{89}$, l'habitude de réfléchir à la composition d'un texte permettant de développer la faculté de choisir l'expression correcte. S'y ajoute la réécriture de textes littéraires latins et, surtout, la traduction de discours grecs qui permettent d'enrichir le vocabulaire de l'orateur et de favoriser la création lexicale ${ }^{90}$. Enfin, l'orateur mémorisera autant de textes que possible ${ }^{91}$. Cet effort quotidien fournira à la fois des réserves où puiser et la capacité de sélectionner le mot juste au moment opportun. De façon subsidiaire, il renforcera les facultés mémorielles, dont les déficiences sont rendues responsables de l'autre type de sécheresse, celui imputable au manque de matière.

Les défaillances de la memoria sont en effet cause de bien des silences et de bien des ridicules : au delà du lexique, c'est sous l'espèce de l'oubli et du trou de mémoire que notre corpus aborde le problème de l'incomplétude du propos. Cicéron évoque, dans le Brutus, la mémoire extrêmement défaillante de l'orateur Caius Scribonius Curio, qui l'empêchait souvent de tenir un discours cohérent et complet faute de se souvenir du plan qu'il s'était fixé. Un jour qu'il affrontait Cicéron, il cessa brutalement de parler car son discours lui avait entièrement échappé :

\begin{abstract}
Memoria autem ita fuit nulla, ut aliquotiens, tria cum proposuisset, aut quartum adderet aut tertium quaereret; qui in iudicio priuato uel maxumo, cum ego pro Titinia Cottae perorauissem, ille contra me pro Ser. Naeuio diceret, subito totam causam oblitus est idque ueneficiis et cantionibus Titiniae factum esse dicebat ${ }^{92}$.
\end{abstract}

Pour ce qui était de la mémoire, Curio en était si dépourvu qu'il lui arrivait, après avoir annoncé trois divisions, d'en ajouter une quatrième ou de ne plus retrouver la troisième. Un jour que dans

88. Cf. Contr. II praef. 4-5.

89. De orat. I, 149-153.

90. De orat. I, 154-155; inst. X, 5, 2-11. L'insistance de Cicéron sur l'enrichissement qu'apporte le travail sur les textes grecs fragilise l'analyse ethnique que Sénèque formule à propos de Cestius.

91. De orat. I, 157-158. Ce travail de mémorisation, au-delà de sa dimension lexicale, a des conséquences proprement culturelles qui contribuent indirectement à la copia : d'après le personnage de Crassus, la richesse des connaissances entraînera naturellement celle de l'expression (de orat. III, 125).

92. Brut. 217 ; trad. J. Martha modifiée. L'expression pro Titinia Cottae perorauissem peut s'interpréter de deux manières (cf. Douglas 1966, p. 158), selon que l'on fait de Cottae un génitif (« femme de Cotta ») ou un datif rapporté à perorauissem (« j'ai clos l'affaire de Titinia dans laquelle Cotta avait parlé avant moi »). Mais la construction d'un datif adjoint à perorare ne se rencontre nulle part ailleurs, et l'on s'accorde généralement à donner au passage le sens de «j'ai plaidé pour la femme de Cotta » (et non «pour la cliente de Cotta » comme le traduit J. Martha) : cf. Malcovati 1965, p. 130 ; Crawford 1984, p. 35, n. 2. Dans l'Orator, Cicéron offre une interprétation différente de ce mutisme et se félicite d'avoir réduit Curion au silence : cf. supra, p. 2. Sur la présence de Curion dans cette affaire, cf. McDermott 1972, p. 395-396 ; sur l'écart entre les deux récits qu'en fait Cicéron, cf. Tatum 1991. 
une cause privée fort importante, j'avais plaidé pour Titinia, la femme de Cotta, son tour étant venu de parler contre moi en faveur de Servius Naevius, il oublia tout à coup tout ce qu'il avait à dire : la faute en était, disait-il, aux philtres et aux sortilèges de Titinia.

Le comique de la situation est évident, et le mutisme de Curion excite le rire ${ }^{93}$. Mais ces silences soudains - partiels quand manque une partie du discours, complet comme dans son plaidoyer pour Naevius - ont des conséquences plus lourdes que cette humiliation passagère, car ils jettent le doute sur ses capacités : on hésitera à lui confier son affaire ${ }^{94}$. Comme Cestius, Curion pousse à la limite le défaut qu'il incarne, la cause de sa faiblesse étant naturelle - elle l'affecte également quand il écrit - plus que technique. Cet orateur amnésique rappelle la nécessité de l'entraînement mémoriel et du développement de la mémoire artificielle ${ }^{95}$.

C'est en structurant sa mémoire par des exercices répétés et en usant d'une méthode efficace $^{96}$ que l'orateur se prémunira contre des oublis de ce genre. Encore faut-il que cette méthode soit appropriée : l'arsenal de la mémoire artificielle doit en effet être manié avec précaution et adapté aux capacités de chacun. Quintilien oppose ainsi deux types de mémorisation, l'une portant sur les mots, l'autre sur les idées et l'organisation qu'on entend leur donner. Sans affirmer la supériorité d'une méthode sur l'autre, ses analyses laissent entendre que la rétention uerbatim est la meilleure lorsque l'on dispose d'une excellente mémoire naturelle ${ }^{97}$, mais que la simple mémorisation des idées doit être privilégiée par ceux qui n'ont pas une mémoire aussi efficace : vouloir à tout prix retenir un texte précis ferait paradoxalement courir le risque du silence. En effet, embarrassé dans un appareil technique qui dépasse et étouffe ses facultés mémorielles, l'orateur peut bafouiller ou, pire encore, rester subitement sec (aut deformem haesitationem aut etiam silentium inducat ${ }^{98}$ ) s'il en vient à n'oublier ne serait-ce qu'un seul mot du discours qu'il a mémorisé et qu'il tente en vain de le retrouver au lieu de développer librement son argumentaire :

La lenteur et la sécheresse - qu'elle soit celle des idées ou du lexique - représentent donc deux manifestations du silence partiel ou complet, et se combattent par une maîtrise technique poussée reposant sur des choix théoriques justes et un long entraînement préalable. À plusieurs reprises, néanmoins, apparaît l'idée que, pour être véritablement efficace, l'orateur doit avoir conscience de ses propres limites (celles de son lexique ou de sa mémoire) pour leur adapter son effort et choisir sa méthode en fonction d'elles. D'après la doctrine, le silence de l'orateur peut parfois être imputable au jugement erroné qu'il porte sur lui-même. De même que la volonté de dépasser ses modèles, l'ambition stylistique peut paralyser l'orateur. Par volonté de bien faire, certains praticiens, souvent des débutants d'après Quintilien, en viennent à se taire par désespoir d'atteindre à la perfection :

\section{Brut. 216.}

94. Brut. 220.

95. Sur la distinction entre mémoires naturelle et artificielle, et les techniques de mémorisation dans la doctrine rhétorique ancienne, cf. Yates 1966, p. 1-26 ; Carruthers 1990, p. 16-45 et récemment Baroin 2010, p. 211 ; Guérin 2011a, p. 708-712 et la bibliographie citée. La doctrine présente fréquemment la memoria comme un facteur essentiel du succès oratoire, cf. de orat. I, 18, 94, 114 ; III, 230 ; Brut. 139, 215, 227, 301, 304.

96. Cf. la méthodologie complète proposée par la Rhétorique à Herennius : Rhet. Her. III, 28-40.

97. C'est dans ce cas de figure que l'orateur, tout en ayant appris son discours, peut le délivrer de manière entièrement naturelle et en donnant l'impression qu'il improvise : cf. Brut. 139.

98. Inst. XI, 2, 48. Voir aussi inst. XI, 6, 7 ; XII, 9, 16-17. 
Accidit enim etiam ingeniosis adulescentibus frequenter, ut labore consumantur et in silentium usque descendant nimia bene dicendi cupiditate ${ }^{99}$.

Il arrive souvent, en effet, et même à des jeunes gens de talent, de s'épuiser au travail et de finir par ne rien dire du tout par un désir excessif de bien s'exprimer.

Le cas est différent de celui de Cestius qui s'arrêtait net alors que les apprentis n'osent pas même commencer à parler, mais le mécanisme qui leur impose le silence est identique. Seule l'évaluation correcte de ses propres capacités permettra à l'orateur de s'exprimer comme il convient, et il est particulièrement dommageable de vouloir parler mieux qu'on ne le peut. Mais il ne faut pas pour autant douter sans cesse de ses capacités, l'orateur ne pouvant atteindre le succès que s'il dispose de la fiducia nécessaire à la parole. Cicéron lui-même soulignait combien il lui était difficile de commencer à parler, l'ampleur de la tâche rendant toujours ses premiers mots hésitants ${ }^{100}$. Mais si cette crainte est salutaire, la confiance de l'orateur en ses propres moyens doit lui permettre de dépasser ce blocage initial. Tout en acceptant le point de vue cicéronien, Quintilien souligne que la uerecundia doit être contrôlée sous peine de tomber dans un subitum silentium et de s'effondrer sous le poids du metus. La fiducia, dans ce cas, n'est pas orgueil mais conscience éclairée de ses capacités :

\footnotetext{
Neque ego rursus nolo eum qui sit dicturus et sollicitum surgere et colore mutari et periculum intellegere, quae si non acciderent, etiam simulanda erant ; sed intellectus hic sit operis, non metus, moueamurque, non concidamus. Optima est autem emendatio uerecundiae fiducia et quamlibet inbecilla frons magna conscientia sustinetur ${ }^{101}$.

Ce n'est pas non plus que je veuille que l'orateur qui s'apprête à parler ne soit pas inquiet en se levant, ne change pas de couleur et ne saisisse pas le risque qu'il court; si l'on n'éprouvait rien, il serait même bon de feindre ; mais que ce soit parce que l'on sent la lourdeur de la tâche, non parce qu'on la redoute et soyons émus, non effondrés. Le meilleur remède à la timidité, c'est la confiance en soi et, quelque défaut d'assurance qu'il présente, l'orateur trouve un soutien dans la conscience de sa grande valeur.
}

En sachant ce qu'il est capable de faire, l'orateur évitera autant le mutisme - en ne visant pas trop haut - que la paralysie - en allant au bout de ce dont il est capable.

À l'impréparation et au manque de fiducia, s'ajoute une dernière cause de mutisme : l'inexpérience. D'après Quintilien, les orateurs novices et les rhéteurs courent le risque d'être frappés d'étonnement et de rester muets face aux réalités du forum ${ }^{102}$. Les contraintes de la pratique ne sont pas celles de l'exercice ou de la déclamation publique : le juge n'intervient pas, l'adversaire interrompt, les erreurs sont immédiatement relevées et le temps imparti est limité. Le premier impératif est donc de s'adapter à ces conditions et de ne pas oublier les enjeux de la situation. Pour avoir négligé le fait qu'il plaidait devant le tribunal des centumvirs et non dans une école, le grand déclamateur Albucius Silus fut contraint au silence. Albucius ayant développé une figure où il acceptait le règlement de l'affaire si son adversaire jurait par les cendres de son père, l'avocat adverse saisit la proposition au vol et accepta de jurer. Le

99. Inst. X, 3, 12 .

100. Cf. par exemple Cic., Deiot. 1 ainsi que le parallèle avec le trac ressenti par Crassus en de orat. I, 120-121.

101. Inst. XII, 5, 4 ; trad. J. Cousin.

102. Inst. XII, 6,5 . 
tribunal trancha immédiatement en sa faveur et Albucius ne put développer davantage son argumentaire : 1'oubli des contraintes judiciaires l'obligea à se taire ${ }^{103}$.

Le second impératif consiste, pour l'orateur, à résister aux perturbations qui émaillent les procès sur un forum qui n'offre pas la tranquillité de l'école : comme le remarque Votienus Montanus, que Sénèque le Père cite dans la préface du livre IX des Controuersiae, les rhéteurs ignorent comment se comporter face aux cris, aux rires ou même au silence du public ${ }^{104}$. L'orateur véritable doit surmonter ces difficultés. Le Brutus ${ }^{105}$ et deux anecdotes de Valère Maxime $^{106}$ fournissent des exemples d'orateurs dont l'autorité et le charisme suffisent à mettre fin aux cris intempestifs du public, mais à la fin de l'ère républicaine, ce mode de régulation est manifestement moins efficace que dans ce passé sans doute largement fantasmé : l'orateur est souvent chahuté, mais doit malgré tout parvenir à faire front et à continuer son discours dans le charivari. Cicéron décrit comment Pompée, le 7 février 56, est parvenu à aller au bout de son discours malgré les hurlements et les injures des hommes de Clodius, obtenant même de temps à autre le silence, et comment Clodius, lorsqu'il prit la parole, fut incapable de montrer la même fermeté :
A. d. VII Id. Febr. Milo adfuit. Dixit Pompeius, siue uoluit. Nam ut surrexit, operae Clodianae clamorem sustulerunt, idque ei perpetua oratione contigit, non modo ut acclamatione sed ut conuicio et maledictis impediretur. Qui ut perorauit (nam in eo sane fortis fuit, non est deterritus, dixit omnia atque interdum etiam silentio cum auctoritate peruicerat) - sed ut perorauit, surrexit Clodius. Ei tantus clamor a nostris (placuerat enim referre gratiam) ut neque mente nec lingua neque ore consisteret ${ }^{107}$.

\begin{abstract}
Le 7 février, Milon comparut. Pompée parla ou du moins il en eut l'intention. Car à peine se fut-il levé que les hommes de Clodius se mirent à pousser des cris, et pendant tout son discours il dut subir une obstruction qui n'était pas faite seulement de clameurs, mais d'invectives et d'injures. Dès qu'il eut achevé (car il montra dans cette occasion un grand courage, il ne se laissa pas démonter, il dit tout ce qu'il avait à dire, et il avait même réussi par moments à poursuivre ses développements dans le silence et avec autorité) - mais quoi qu'il en soit, à peine eût-il achevé que Clodius bondit à la tribune. De tels cris l'accueillirent (car les nôtres avaient voulu lui rendre la politesse) qu'il ne fut plus maître de sa pensée, de son langage, de sa physionomie.
\end{abstract}

Parler devant le public romain suppose donc, dans des cas d'agitation extrême, d'imposer sa parole. Puisque réduire l'adversaire au silence représente une forme de victoire suprême, la résistance au chahut constitue à l'évidence un premier signe de puissance oratoire. Si la doctrine ne fournit pas de moyen permettant d'atteindre ce résultat, on perçoit que cette capacité dépend également de la fiducia, de l'expérience mais aussi de l'ampleur et de l'autorité politiques.

103. Contr. VII praef. 7. Voir aussi Inst. orat. IX, 2, 95 ; Suet., gram. 30, 5. Sur cet épisode, cf. Fairweather 1981, p. 141-142 ; Balbo 2007, p. 381-382 ; Berti 2007, p. 144-149.

104. Contr. IX praef. 4.

105. Brut. 54-56.

106. Val. Max. III, 7,3 ; VI, 2, 3 .

107. Ad Q.fr. II, 3, 2 ; trad. J. Beaujeu modifiée. Cf. également fam. I, 5a, 1 qui rapporte plus brièvement la même anecdote, et Att. I, 16, 10 où Cicéron décrit comment, lors d'un échange avec Clodius à la suite du procès de celuici (en 61 av. J.-C.), les sénateurs firent taire son adversaire en le huant (magnis clamoribus adfectus conticuit et concidit). 
L'analyse de ces trois causes du silence - impréparation, état d'esprit, inexpérience - ouvre par ailleurs des possibilités nombreuses dans la critique des théories concurrentes, parfois accusées de pousser l'orateur au mutisme. Dans la lutte qui l'oppose aux Atticistes, Cicéron avance ainsi que la sécheresse stylistique de ses adversaires n'est pas éloignée du silence ou, à tout le moins, de l'inopia ${ }^{108}$. De la même manière, sa polémique contre l'éloquence stoïcienne ${ }^{109}$ amène Cicéron à critiquer l'incapacité oratoire supposée des stoïciens ${ }^{110}$ et à présenter les traités rhétoriques produits par Cléanthe et Chrysippe comme des textes destinés à ceux qui souhaitent garder le silence ${ }^{111}$. Un tel usage polémique n'a rien de surprenant, et ces attaques n'enrichissent pas la compréhension du silence oratoire. Elles se contentent de faire fond sur les catégories que nous avons évoquées pour développer des arguments dans la lutte pour la définition du meilleur style. À l'inverse, il est une absence qui doit retenir l'attention dans cette description technique et étiologique : celle des interprétations du silence rencontrées dans les discours eux-mêmes, qui ne sont nulle part prises en compte par la doctrine. La rhétorique n'analyse jamais les blocages que les discours attribuent à la force de la vérité, à la culpabilité ou à la prévarication. Ces silences relevant du fait brut et non de la technique, la théorie n'a pas à détailler ces interprétations pour elles-mêmes. Plus encore, les analyses techniques du silence tendent à les évacuer. La prévarication et la mauvaise conscience n'interviennent pas dans un corpus qui suppose bien sûr la moralité de l'orateur ; quant à la question de la vérité, elle n'est pas centrale dans le contexte de la persuasion qui s'intéresse uniquement au crédible et pour qui l'orateur réduit au mutisme par l'argument d'un adversaire est moins confondu par le vrai que mal préparé. En reliant la lutte contre le silence à la maîtrise technique, à l'entraînement, à l'expérience et à la fiducia, la doctrine laisse entendre que le véritable orateur ne peut être réduit au silence par une cause extérieure, mais qu'il l'est toujours par sa propre faute.

\section{La machine à discours}

Reste que la préparation, aussi complète qu'elle soit, peut être prise en défaut. Si l'on suppose l'orateur physiquement et intellectuellement apte à accomplir sa tâche, mais également bien préparé techniquement et doté d'une solide expérience, on peut certes considérer que les principaux risques de mutisme ont été éliminés. Pourtant, l'imprévu peut toujours surgir, et le silence causé par la surprise venir ruiner l'ensemble du discours - de façon d'autant plus dommageable qu'il sera interprété comme un mutisme causé par la ueritas. Il est essentiel que l'orateur limite le risque de l'imprévu autant qu'il le peut. Quintilien conseille ainsi d'interroger le client sans ménagement, car celui-ci a tendance à dissimuler et à mentir ${ }^{12}$ : l'orateur apprend parfois de la bouche de son adversaire un point essentiel de l'affaire qu'il ignorait, et voit s'effondrer tout l'édifice qu'il avait construit ${ }^{113}$. Mais il est des cas où la

108. Sur la polémique opposant Cicéron et les Atticistes, cf. Guérin 2011, p. 339-349 et la bibliographie fournie. 109. Cf. Atherton 1988 ; Lévy 2000.

110. Brut. 118.

111. Fin. IV, 7.

112. Inst. XII, 8, 9-11.

113. Inst. XII, 8, 6. 
surprise survient malgré tout - procès anticipé, incident, nécessité de répondre à une réfutation impossible à prévoir ${ }^{114}$ - et où se fait à nouveau sentir le danger du silence.

Face à cette menace, il est possible de lire certains passages de la doctrine élaborée par Quintilien non plus comme une rhétorique ambitieuse visant à donner les moyens de persuader, mais comme une méthode plus modeste évitant à l'orateur d'être réduit au silence dans des circonstances difficiles : si l'orateur est seul responsable de son mutisme, il appartient à la théorie de lui fournir les moyens de réagir dans l'urgence. On pourrait opposer à la démarche de Quintilien que, quelle que soit la version de la doctrine à laquelle on s'intéresse, l'inuentio représente un outil permettant de produire de la matière et donc de lutter contre la sécheresse. De fait, les topiques et les moules de présentation, les amplifications et les généralisations, les exempla préconstitués sont autant d'instruments permettant une réaction rapide et la dissimulation d'un trou de mémoire ou du trouble causé par une objection. On croiserait là l'un de modes de fonctionnement de la « langue de bois » contemporaine, qui sert tout autant à masquer ce qu'on ne veut pas dire et qui embarrasse, qu'à dissimuler l'incapacité à répondre à une question faute de matière ${ }^{115}$. Mais dans ce cas, l'outil servant à éviter le silence est un pis aller. Les topiques et les amplifications demandent en effet une longue réflexion préalable sous peine de tomber à plat, d'être excessivement banales ou de dénoncer leur nature réelle : la langue de bois ne devient ce qu'elle est que parce qu'elle est perceptible par le public.

À l'inverse, il semble possible d'identifier dans l'Institution oratoire une méthode destinée à lutter contre le mutisme sans constituer pour autant une utilisation pauvre d'éléments qui n'étaient à l'origine pas conçus dans ce but. Les développements que Quintilien consacre à l'improvisation - les discours délivrés ex tempore - dessinent en effet le fantasme d'un orateur devenu véritable « machine à discours », impossible à prendre en défaut et capable de s'exprimer en toutes circonstances. Car parler ex tempore ne signifie pas, pour Quintilien, prononcer les mots qui nous viennent à l'esprit afin de combler un vide ${ }^{116}$, mais construire un discours, le retenir, le contrôler et le délivrer dans un seul mouvement. L'improvisation se conçoit comme une composition dont la cogitatio et la dictio sont extrêmement rapprochées, et donc comme un processus où toutes les tâches de l'orateur, habituellement distinctes, sont accomplies d'un seul mouvement :

[...] uix tamen aut natura aut ratio in tam multiplex officium diducere animum queat ut inuentioni, dispositioni, elocutioni, ordini rerum uerborumque, tum iis quae dicit, quae subiuncturus est, quae ultra spectanda sunt, adhibita uocis pronuntiationis gestus obseruatione una sufficiat. Longe enim praecedat oportet intentio ac prae se res agat, quantumque dicendo consumitur, tantum ex ultimo prorogetur, ut, donec perueniamus ad finem, non minus prospectu procedamus quam gradu, si non intersistentes offensantesque breuia illa atque concisa singultantium modo eiecturi sumus ${ }^{117}$.

[...] c'est à peine si la nature ou la méthode pourrait diviser l'esprit entre des fonctions aussi multiples : suffire en même temps à l'invention, à la disposition, à l'élocution, à l'arrangement des idées et des mots, puis à ce que l'on dit, à ce que l'on va ajouter, à ce qu'il faut voir au-delà tout en ayant observé les principes qui règlent la voix, le débit, le geste. Il faut en effet que l'attention se porte loin en avant et qu'elle fasse lever les idées qui sont devant elle et, à mesure que le fonds

114. Inst. VII, 2-3.

115. Sur les pratiques politiques et médiatiques contemporaines de «la langue de bois », cf. Delporte 2009.

116. Inst. X, 7, 12.

117. Inst. X, 7, 8-10 ; trad. J. Cousin modifiée. 
s'épuise avec la parole, que l'orateur fasse l'avance sur ses fonds de réserve, pour que, tant que nous ne sommes pas parvenus à la conclusion, le regard n'avance pas moins que l'allure, si nous voulons éviter de tomber en panne, de broncher ou d'émettre des phrases brève et entrecoupées comme ceux qui parlent en sanglotant.

Ce qui était interdit dans le cadre de la préparation normale du discours ${ }^{118}$ devient ici le moyen même du succès, avant tout parce que l'orateur s'est formé à cet exercice périlleux.

Le but de la méthode proposée par Quintilien est triple : éviter la sécheresse, mais aussi conserver une structure solide au discours et empêcher tout débordement où l'orateur développerait son propos sans limite pour masquer l'absence de matière et la panique dans laquelle il se trouve ${ }^{119}$. Dans les trois cas, on retrouve une modalité du silence oratoire, qu'il soit silence complet, parole erratique ou discours tendant vers le creux. C'est la stricte organisation du propos qui permettra l'enchaînement naturel des idées, la production d'un discours qui ne sera pas entrecoupé - l'orateur sachant quoi dire dans chaque partie - et le respect de la mesure dans le développement ${ }^{120}$ : l'improvisation suppose une dispositio plus rigide encore que dans un discours préparé. Elle exige également un débit plus lent qu'à l'habitude - précisément pour laisser le temps à la réflexion de se développer et éviter une diction chaotique - ainsi qu'un certain oubli des impératifs stylistiques pour se concentrer sur le fond ${ }^{121}$ : le but est de contrôler la parole et non de se laisser porter par elle. Cette méthode libérera l'esprit de l'orateur pour lui permettre de construire ses arguments et de les délivrer dans un délai très rapproché.

La mise en œuvre accélérée de processus intellectuels aussi divers n'est bien sûr rendue possible que par une rétention mémorielle à court terme ${ }^{122}$ devenue efficace à force d'entraînement, et par véritable souplesse intellectuelle (mobilitas animi ${ }^{123}$ ). L'improvisation est en premier lieu un exercice de remémoration, et c'est bien la memoria qui représente la protection la plus sûre contre le silence. Si les techniques mémorielles interviennent, c'est une fois encore la nature de l'orateur qui conditionnera sa facultas ex tempore dicendi, l'exercice supposant une mémoire naturellement sûre et prompte. Tout au long de son discours improvisé, l'orateur pourra ainsi construire une réserve argumentative dans laquelle il puisera au fur et à mesure et qu'il remplira à nouveau tout en parlant ${ }^{124}$. Dans ces circonstances, les orateurs minus prompti seront désavantagés ${ }^{125}$, la doctrine rapprochant à nouveau, quoique de manière implicite, silence et stupidité.

Directement dépendante de la nature de l'orateur et de la méthode qu'il met en œuvre, la facultas ex tempore dicendi demeure malgré tout le fruit du labor et du studium ${ }^{126}$. La doctrine

118. Quintilien souligne néanmoins que la capacité d'improvisation ne doit pas amener l'orateur à multiplier les causes et à négliger sa préparation (inst. X, 7, 20-21). Quintilien expose sa méthode <non> ut ex tempore dicere malit, sed ut possit (ibid. 4).

119. Inst. X, 7, 13 .

120. Inst. X, 7, 5-7.

121. Inst. X, 7, 14, 22.

122. Sur l'usage de la mémoire à court terme dans les pratiques d'improvisation, Cf. Guérin 2011a, p. 715-716.

123. Inst. X, 7, 8. Cf. également Inst. V, 10, 123.

124. Inst. X, 7, 10, cité supra p. 19.

125. Inst. XII, 9, 16.

126. Inst. X, 7, 1. On retrouve ici l'idée selon laquelle la préparation, de manière générale, offre une garantie 
de l'improvisation élaborée par Quintilien est très proche des préceptes censés garantir la copia que nous avons déjà rencontrés. Ainsi, une pratique continue de la lecture et de l'écriture fournira à l'orateur la matière et le lexique nécessaires, mais aussi les automatismes et les développements prêts à l'emploi qui lui permettront d'atteindre la facilitas dans l'improvisation ${ }^{127}$. Cette facultas se forge par des entraînements quotidiens - car elle se perd aussi vite qu'elle s'acquiert ${ }^{128}$ : c'est en improvisant en silence des discours entiers que l'on développera sa capacité à réagir face à l'imprévu ${ }^{129}$.

La doctrine de l'improvisation élaborée par Quintilien ne représente donc pas une simple recension de topiques ou de moules d'invention permettant de répondre à l'urgence, mais suppose un usage spécifique des différentes composantes de la technique orientée vers un but unique : éviter le mutisme. De ce fait, Quintilien reconfigure la méthode d'invention normale en demandant à l'orateur une mobilisation bien particulière des facultés qu'il emploie habituellement. Ainsi doté et préparé, l'orateur fera preuve d'une attention permanente à ce qui l'entoure et aux arguments de l'adversaire, et sera toujours prêt à abandonner ce qu'il a préparé pour se lancer dans un développement improvisé qui réponde aux exigences du moment. La pire réaction serait de se raccrocher à ce qui avait été écrit et préparé pour tenter de l'adapter : c'est là le meilleur moyen d'être excessivement ralenti dans son propos ou d'être court ${ }^{130}$.

Quintilien propose ainsi une adaptation des techniques normales d'inuentio afin de contrer le défaut du mutisme. L'orateur préparé et entraîné ne doit en aucun cas se laisser surprendre ou refuser de prendre la parole ${ }^{131}$. La raison de cet interdit est profonde. Car si, pour Quintilien, le silence causé par l'imprévu est inacceptable, il l'est moins pour des raisons stratégiques que pour des raisons éthiques. D'après lui, en effet, l'orateur frappé par le silence est non seulement disqualifié d'un point de vue technique, mais aussi d'un point de vue moral :

Vix enim bonae fidei uiro conuenit auxilium in publicum polliceri quod praesentissimis quibusque periculis desit, [...] siquidem innumerabiles accidunt subitae necessitates uel apud magistratus uel repraesentatis iudiciis continuo agendi. Quarum si qua, non dico cuicumque innocentium ciuium, sed amicorum ac propinquorum alicui euenerit, stabitne mutus et salutarem petentibus uocem statimque, si non succurratur, perituris moras et secessum et silentium quaeret, dum illa uerba fabricentur et memoriae insidant et uox ac latus praeparetur ${ }^{132}$ ?

Car il ne convient guère à un homme de bonne foi de promettre à tous une assistance qui pourrait faire défaut dans les dangers les plus pressants, [...] puisque se présentent d'innombrables nécessités imprévues où il faut plaider immédiatement, soit devant les magistrats, soit dans des procès appelés par anticipation. Si l'une de ces éventualités se présente, je ne dis pas pour un citoyen innocent quelconque, mais pour l'un de ses amis ou de ses proches, l'orateur restera-t-il debout et muet et, lorsqu'ils attendent de lui une parole salvatrice et qu'ils vont succomber si on ne leur porte pas secours, demandera-t-il un délai, l'isolement et le silence pour avoir le temps de forger ces belles paroles, de les fixer dans sa mémoire et de préparer sa voix et ses poumons ?

contre le silence.

127. Inst. X, 7, 7-8, 28-29.

128. Inst. X, 7, 24, 27.

129. Inst. X, 7, 25.

130. Inst. XII, 9, 18.

131. Inst. XII, 9, 20-21.

132. Inst. X, 7, 1-2 ; trad. J. Cousin modifiée. 
L'obligation d'assistance faite au patronus qui prend en charge l'affaire de son client lui interdit de se taire : l'accomplissement de sa fides et de sa diligentia exige sinon la victoire, du moins un discours correct marquant un engagement suffisant dans la lutte ${ }^{133}$. L'orateur véritable doit toujours être capable de prendre la parole, quelles que soient les circonstances ${ }^{134}$ : on n'est pas aliquando orator, orateur de temps à autre, on l'est en permanence ou jamais ${ }^{135}$. Ainsi, c'est avant tout comme l'instrument d'une éthique oratoire fondée sur l'interdit du silence qu'il faut interpréter la technique d'improvisation qu'élabore Quintilien.

\section{Conclusion}

Le silence de l'orateur appelle deux analyses bien distinctes selon qu'on se situe dans le domaine de la pratique ou dans celui de la théorie. À l'interprétation du silence considéré comme un signe d'embarras, de culpabilité ou de malhonnêteté, s'oppose l'analyse du silence comme défaut à supprimer ou à masquer. Est-ce à dire que l'orateur doté d'une bonne natura, d'une vaste expérience et d'une parfaite maîtrise de la technique, mais aussi d'un entrâ̂nement spécifique à l'improvisation, échappera systématiquement au danger du mutisme ? D'un point de vue pratique, la réponse est évidemment négative - il suffit de penser aux mésaventures de Cicéron lui-même pour s'en convaincre ${ }^{136}$ - et la théorie, même lorsqu'elle propose une description idéalisée de l'orateur, ne prétend d'ailleurs pas cela. Il existe en effet un accident qui contraindra nécessairement l'orateur au silence, aussi habile et préparé qu'il soit : la disparition du public.

Lorsqu'il évoque dans le Brutus le cas de C. Scribonius Curio, consul en 76, qui fut un jour abandonné par le public au beau milieu de son discours, Cicéron demande à Brutus quelle serait sa réaction dans une situation de ce genre :

Quid tu, Brute? possesne, si te ut Curionem quondam, contio reliquisset? Ego uero, inquit ille, ut me tibi indicem, in eis etiam causis, in quibus omnis res nobis cum iudicibus est, non cum populo, tamen si a corona relictus sim, non queam dicere ${ }^{137}$.

Et toi, Brutus, pourrais-tu dire un mot si tu te voyais, comme le fut un jour Curion, abandonné par le public d'une assemblée ? Je te l'avouerai sans détour, dit-il : même dans les affaires qui dépendent uniquement des juges et où la foule n'a rien à voir, si l'assistance venait à me laisser là, je serais incapable de parler.

La remarque qui précède ce passage révèle toute la spécificité de la situation oratoire : si Antimaque a pu lire son poème quand tous l'avaient abandonné à l'exception de Platon, Démosthène lui-même, nous dit Cicéron, n'aurait pu prononcer un seul mot devant une assemblée vide ${ }^{138}$. L'orateur ne peut parler sans public.

Le constat pourrait paraître naîf ; il doit s'interpréter à la lumière des implications politiques du Brutus et, en particulier, des critiques que l'ouvrage adresse à César pour avoir réduit le

133. Sur cette obligation d'assistance et d'engagement, cf. supra, p. 1, n. 3 .

134. Cf. l'exemple de Galba, décrit en Brut. 87, qui se charge d'une cause complexe, ne dispose que d'une journée pour la préparer, la plaide et remporte la victoire.

135. Inst. X, 7,3 .

136. Cf. sur ce point les commentaires d'Asconius au Pro Milone de Cicéron (Mil. 42C) et les références fournies par Lewis 2006, p. 247.

137. Brut. 192 ; trad. J. Martha modifiée. Il s'agit ici encore de Curio Pater, l'orateur à la mémoire déficiente cité en Brut. 217 et en orat. 129.

138. Brut. 191. 
forum au silence ${ }^{139}$. En 46 av. J.-C., le risque de l'abandon ne menace plus seulement le mauvais orateur mais bien l'éloquence dans son entier : quel destin sera réservé à l'art oratoire dans une cité où la parole est monopolisée par un seul ? À cette nouvelle forme de mutisme le silence imposé par le pouvoir politique -, le Brutus oppose plusieurs réactions possibles : sauvegarde de la pratique oratoire par un cercle qui saura la perpétuer ${ }^{140}$, transformation de l'éloquence par l'échange écrit ${ }^{141}$ ou à travers l'écriture de l'histoire ${ }^{142}$ par exemple. Pourtant, aucune de ces possibilités n'est véritablement satisfaisante : l'éloquence passe avant tout par une voix, s'incarne dans un corps et nécessite, pour exister pleinement, les réactions d'une foule. C'est là le sens véritable de la situation qu'imagine Cicéron lorsqu'il décrit l'orateur privé de public ou qu'il déplore la mort de l'éloquence ${ }^{143}$ : au delà de la technique, de l'exercice et de ses qualités naturelles, c'est avant tout de conditions politiques favorables soit, dans l'esprit de Cicéron, des institutions républicaines - que l'orateur a besoin pour vaincre le silence.

\section{Bibliographie}

Aldrete G. S., 1999, Gestures and Acclamations in Ancient Rome, Baltimore, John Hopkins University Press.

Alexander M., 1976, « Hortensius' Speech in Defense of Verres », Phoenix, 30, p. 46-53.

Atherton C., 1988, « Hand Over Fist : The Failure of Stoic Rhetoric », CQ, 38, p. 392-427.

Baker R. R., 1982, « Sallustian silence », Latomus, 41, p. 801-802.

Balbo A., 2007, « Alcuni casi di interazione oratore-pubblico a Roma tra il I secolo a.C. e il I d.C », $C C G, 18$, p. 375-388.

Bardon H., 1943, « Le silence, moyen d'expression », REL, 21-22, p. 102-120.

Baroin C., 2010, Se souvenir à Rome : formes, représentations et pratiques de la mémoire, Paris, Belin.

Baroin C. 2010a, « Intégrité du corps, maladie, mutilation et exclusion chez les magistrats et les sénateurs romains ", in F. Collard et É. Samama éd., Handicaps et sociétés dans l'histoire. L'estropié, l'aveugle et le paralytique de l'Antiquité aux temps modernes, Paris, L'Harmattan, p. 49-68.

Berti E., 2007, Scholasticorum studia. Seneca il Vecchio e la cultura retorica e letteraria della prima età imperiale, Pise, Giardini.

Carter L. B., 1986, The Quiet Athenian, Oxford, Clarendon Press.

Corbeill A., 2002, « Ciceronian Invective, », in J. May éd., Brill's Companion to Cicero. Oratory and Rhetoric, Leiden, Brill, p. 197-217.

Crawford J.W. (éd.), 1984, M. Tullius Cicero : the Lost and Unpublished Orations, Göttingen Vandenhoeck \& Ruprecht.

Dalimier C., 1989, « L'aposiôpèse selon Quintilien : une timide réflexion sur le sens du

139. Brut. 6, 324, 332. Cf. également off. II, 3. César lui-même est présenté par Cicéron comme une figure qui, par son style propre, ruine les possibilités d'interaction et impose le silence à autrui : cf. sur ce point l'analyse de Gurd 2012, p. 58-66.

140. Brut. 23, 330 .

141. Gurd 2012, p. 70-71.

142. Gowing 2000 ; Steel 2003.

143. Brut. 1-6, 22, 329-333. 
silence ", in Rhétorique et discours critiques. Échanges entre langue et métalangues, Actes du colloque tenu à l'École normale supérieure les 13 et 14 mars 1987, Paris, Presses de l'École normale supérieure, p. 27-32.

David J.-M. 1986, « La faute et l'abandon. Théories et pratiques judiciaires à Rome à la fin de la République », in L'aveu. Antiquité et Moyen Âge. Actes de la table ronde de Rome (28-30 mars 1984), Rome, École Française de Rome, p. 71-87.

David J.-M., 1992, Le patronat judiciaire au dernier siècle de la république romaine, Rome, École Française de Rome.

DeLacy P., 1941, « Cicero's Invective against Piso », TAPhA, 72, p. 49-58.

Delporte C., 2009, Une histoire de la langue de bois, Paris, Flammarion.

Douglas A.E. (éd.), 1966, M. Tulli Ciceronis Brutus, Oxford, Clarendon Press.

Ducos C. 2010, "Penser et surmonter le handicap : les écrits des juristes romains », in F. Collard et É. Samama éd., Handicaps et sociétés dans l'histoire. L'estropié, l'aveugle et le paralytique de l'Antiquité aux temps modernes, Paris, L'Harmattan, p. 69-83.

Elissalde Y., 1997, Du silence : l'homme et ses prosopopées, Bordeaux, Presses universitaires de Bordeaux.

Fairweather J., 1981, Seneca the Elder, Cambridge, Cambridge University Press.

Gardner J. F., 2002, Being a Roman Citizen, Londres, Routledge.

Garland R., 1995, The Eye of the Beholder : Deformity and Disability in the Graeco-Roman World, Duckworth, Londres.

Glenn C., 2004, Unspoken : a Rhetoric of Silence, Carbondale, Southern Illinois University Press.

Gowing A. M., 2000, « Memory and Silence in Cicero's Brutus », Eranos, 98, p. 39-64.

Guérin C., 2009, Persona. L'élaboration d'une notion rhétorique au I ${ }^{e r}$ siècle av. J.-C. Volume $I$ : antécédents grecs et première rhétorique latine, Paris, Vrin.

Guérin C., 2009a, «Frangere aduersarium : usages et limites de la violence oratoire dans la rhétorique cicéronienne, », in V. Azoulay et P. Boucheron éd., Le mot qui tue. Une histoire des violences intellectuelles de l'Antiquité à nos jours, Paris, Champ Vallon, p. 225-240.

Guérin C., 2011, Persona. L'élaboration d'une notion rhétorique au I ${ }^{\text {er }}$ siècle av. J.-C. Volume II : théorisation cicéronienne de la persona oratoire, Paris, Vrin.

Guérin C., 2011a, « La tablette et la torche : mémoire, composition et performance oratoire dans la rhétorique romaine, », in C. Jacob éd., Les lieux de savoir II, Paris, Albin Michel, p. 723-743.

Gurd S., 2012, Work in Progress. Literary Revision as Social Performance in Ancient Rome, Oxford, Oxford University Press.

Le Goff J., 1989, « Rire au Moyen Âge », Cahiers du CRH, 3, p. 1-14.

Levene D. S., 2004, « Tacitus' Dialogus As Literary History », TAPhA, 134, p. 157-200.

Lévy C., 2000, « Cicéron critique de l'éloquence stoïcienne », in L. Montefusco-Calboli éd., Papers on Rhetoric III, Bologna, CLUEB, p. 127-144.

Lévy C., 2006, « La notion de color dans la rhétorique latine : Cicéron, Sénèque le Rhéteur, Quintilien, », in A. Rouveret, V. Naas et S. Dubel éd., Couleurs et matières, Paris, Éditions Rue d'Ulm, p. 185-99.

Lewis R. G. et al. éd., 2006, Asconius Pedianus, Commentaries on Speeches of Cicero, Oxford, Oxford University Press.

Malcovati E., 1968, « Sulle ultime edizione del Brutus », Athenaeum, 46, p. 122-130. 
Mazzeo J. J., 1962, « St. Augustine's Rhetoric of Silence », JHI, 23, p. 175-196.

McDermott W. C., 1972, « Curio Pater and Cicero », AJPh, 93, p. 381-411.

Mommsen T., 1892, Le droit public romain. Tome II, traduit par P. F. Girard, Paris, Ernest Thorin.

Montefusco-Calboli L., 2003, «Ductus and Color : The Right Way to Compose a Suitable Speech », Rhetorica, 21, p. 113-131.

Montiglio S., 2000, Silence in the Land of Logos, Princeton, Princeton University Press.

Moreau P., 2003, « Donner la parole au peuple ? Rhétorique et manipulation des contiones à la fin de la République romaine », in S. Bonnafous, P. Chiron, D. Ducard et C. Lévy éd., Argumentation et discours politique. Antiquité grecque et latine, Révolution française, Monde contemporain. Actes du colloque international de Cerisy-la-Salle, Rennes, Presses universitaires de Rennes, p. 175-89.

Osgood J., 2007, « The Vox and Verba of an Emperor : Claudius, Seneca and Le Prince Ideal », CJ, 102, p. 329-353.

Perelman C. et Olbrechts-Tyteca L., 1958, La nouvelle rhétorique. Traité de l'argumentation, Paris, Presses universitaires de France.

Schenkeveld D. M., 1988, « Iudicia vulgi : Cicero, De oratore 3, $195 \mathrm{ff}$. and Brutus $183 \mathrm{ff}$ », Rhetorica, 6, p. 291-305.

Steck U., 2009, Der Zeugenbeweis in den Gerichtsreden Ciceros, Francfort-sur-le-main, Peter Lang.

Steel C. E. W., 2003, « Cicero's Brutus : The End of Oratory and the Beginning of History ? », BICS, 46, p. 194-211.

Tatum W. J., 1991, " Cicero, the Elder Curio, and the Titinia Case », Mnemosyne, 44, p. 364-371.

Thomas J.-F., 2007, Déshonneur et honte en latin :étude sémantique, Louvain, Peeters.

Valette-Cagnac E., 2004, « La figure de la reticentia dans les traités de rhétorique et dans la pratique judiciaire de Cicéron », in L. Louvel et C. Rannoux éd., La réticence, La Licorne, Rennes, Presses universitaires de Rennes, p. 101-121.

Woodman A. A., 1973, « A Note on Sallust, Catilina 1.1 », CQ, 23, p. 301. 